\title{
What do you do after you say hello? Observing, coding, and analyzing parent-infant interactions
}

\author{
DOUGLAS B. SAWIN, JUDITH H. LANGLOIS, and EDWARD F. LEITNER \\ University of Texas at Austin, Austin, Texas 78712
}

\begin{abstract}
Procedures for observing, coding, and analyzing parent-infant interactions are described. Sample data are presented to illustrate the outcome of these procedures and the manner in which developmental issues can be addressed using the hardware and software systems described.
\end{abstract}

The purpose of this paper is to describe our use of the Datamyte Model 900 series electronic event recorder for the collection of behavioral data in naturalistic observations of parent-infant interactions. The primary focus of our current research is to assess styles of parenting and infant development over the first year of infant life. From observing and recording parent-infant dyadic interactions, we derive four types of data: (1) the frequencies of discrete behavioral events (e.g., touch, pat); (2) the durations of continuous behavioral events (e.g., smile); (3) sequences or patterns of individual behaviors (e.g., infant spits up $\rightarrow$ infant cries) and interactions between members of dyads (e.g., infant spits up $\rightarrow$ parent looks more closely $\rightarrow$ adjusts infant's position $\rightarrow$ infant stops crying); (4) conditional probability estimates of the contingencies between behaviors of one member of the dyad and those of the other member. Standard statistical techniques (e.g., ANOVA, MANOVA, correlational) are employed to make group comparisons and to establish individualdifference characteristics of parents and infants for these types of behavior variables. Two global characteristics of behavior patterns in parent-infant interactions are distinguished: (1) quantitative aspects such as amount of stimulation and affection provided for the infant by the parent (determined by total frequencies and durations of behaviors and sequences of behaviors) and (2) qualitative aspects such as parental sensitivity and responsiveness to infant cues (based on the contingencies between infant behaviors and parent responses), and vice versa.

Our current work involves the longitudinal assessment of parent-infant interactions in a multiethnic sample (Black, Mexican-American, and Anglo-American)

The authors wish to express their appreciation to Chris Downs, Darryl Gowan, Debby Harris, Linda King, Martha Lillie, and Cathy Radek for their outstanding assistance in the data collection and analysis for this project. Requests for reprints should be addressed to Douglas Sawin, Department of Psychology, University of Texas, Austin, Texas 78712. of mothers, fathers, and their infants. By means of analyses of variance of frequencies, durations, and conditional probabilities, comparisons of patterns of parentinfant interaction for mothers vs. fathers can be made, as well as comparisons based on age of the parents, sex and/or age of infant, ethnic group, socioeconomic status, and individual-difference variables (e.g., parent and infant temperaments). Issues addressed include the modifying influence of both parent and infant characteristics in the dyad, changes in the behavior of parents and infants over time due to parent experience and infant age, adaptation of parents to emerging infant capacities and behaviors, and the effects of different patterns of early parenting on subsequent infant cognitive and social development. Analyses involving conditional probabilities permit the assessment of the direction of effects of behavior. Cross-lag-panel analyses of the correlational data across time permit causal inferences to be made about the impact of parent and infant on each other over development.

\section{OBSERVING INTERACTIONS}

The Datamyte 900 (or 901), a portable hand-held battery-operated event recorder, performs three basic functions in our research: (1) data collection, (2) shortterm storage, and (3) data interface with central computer facilities (see Torgerson, 1977, for a more complete description of the Datamyte system).

The behavioral events to be sampled are assigned code numbers prior to data collection. During the observation sessions, each behavioral event-is recorded by punching in its designated code number on the keyboard of the Datamyte and then pressing the "enter" switch. At the time the "enter" switch is pressed, three pieces of datum are stored: (1) the line number of the behavior code being entered, which indicates the order and the cumulative total number of codes entered; (2) the code number of the observed behavior; (3) the time of occurrence of the entered behavior code, indicated in time elapsed since the onset of the observation session. The 
time of occurrence record for each entry allows time lag analyses and permits the post hoc establishment of time intervals for the determination of temporal contingencies between events. Thus, the behavior record that is stored and later retrieved consists of a list of behavior code numbers in the sequence in which they were entered. The order of the entered code is indicated by a cumulative numerical index, and the time of each entered code is indicated in hurdredths of a minute of time elapsed.

The Datamyte allows the user to preserve and retrieve the exact sequence of the behavior codes as entered by the observer. Most important for our purposes is the capacity of the system to record and store the entries on a continuous real-time line. This allows the exact specification of the temporal relation (e.g., time lag) between any two entries and the precise duration between a code number indicating the onset of a continuous event and the code number indicating the termination of that event.

Providing that the coding system utilizes an additional digit for each behavior code to indicate the actor and/or object of a behavior, one observer is able to record the behaviors of two or more subjects at the same time. With the Datamyte system, the entry of events is quick, taking only as long as the time to press the number of keys prescribed by the numerical code for that event.

Since Datamyte units are battery operated, batteries must be charged and checked at regular intervals. Data loss is the painful consequence of neglected batteries. As with any electronic/mechanical equipment, Datamyte units are susceptible to malfunction and failure. Problems were very common with older model units from the same manufacturer (e.g., DAK 8) which utilized a cassette tape recorder for storage. The newer solid state memory units are more reliable. Our experience with two units over the past year involved only one failure resulting in data loss. The problem in that case was quickly remedied by the manufacturer. The company representative willingly and expediently provided us with a "loaner" during the repair period.

The Datamyte event recording system is considerably more expensive than a paper and pencil scoring and coding system. However, the expense involved in purchase and maintenance of Datamyte is generally not greater than video equipment for the same purpose, and may be less, in the long run, than the personnel costs of data management done by hand.

\section{Observers}

Observers must memorize a code number for each behavioral event to be sampled. Thus, a disadvantage of this system is that, for large comprehensive coding systems, the training of observers takes longer than would be necessary with paper and pencil techniques where observers can use acronyms or symbols that have inherent meaning. The translation of behavioral events into less meaningful code numbers, pressing the correct Datamyte keys in the correct order, and doing this rapidly require highly skilled and thoroughly trained personnel. A multistage 4to 6-week training period is required to achieve acceptable levels of interobserver reliability with our coding system, which includes over 60 parent behavior and 60 infant codes. Even with highly skilled and highly trained observers, errors due to pressing the wrong keys occur occasionally and must be subsequently searched for and identified by an error program and by careful review of the printout of behavior records. The errors must be corrected on the computer magnetic tape files by subsequent editing of the behavior records. This process is quite time consuming. In our research, approximately $3 \mathrm{~h} /$ observation are required for all phases of editing and data preparation for data analysis. Of course, errors that are incorrect because one legal code number has been substituted for another cannot usually be detected by the error program or by "eyeballing" and can only be ascertained by reliability checks.

\section{OBSERVATIONAL PROCEDURES}

We are interested in establishing frequencies and durations of behavior, patterns of interaction, and the conditional probability estimates of the temporal contingencies between behavioral events for molecularly defined behaviors observed in parent-infant interactions. Mother-infant and father-infant dyads are observed during interactions in the hospital within $72 \mathrm{~h}$ following birth of the infant and at successive 3-month time points through the first year. At the hospital and during the 3-month measurement times, parent-infant dyads are observed for 10-min feeding and 10-min playing sessions. The observer, holding the Datamyte unit, is present in the room with the parent-infant pair, but does not interact with them.

\section{CODING INTERACTIONS}

We use five caregiver-infant interaction behavior coding systems. The five differ only in minor modifications (additions and deletions of specific behaviors) that reflect the relevance of specific parent and infant behaviors for different time points of infant development during the first year. The behavior coding systems include approximately 60 parent behaviors and 60 infant behaviors, each of which is represented by a four-digit code number. ${ }^{1}$ The first digit indicates the actor, the second digit indicates the general class of behaviors to which the specific observed behavior belongs (e.g., holding positions, feeding activities, visual activities, tactual stimulation); the third indicates the behavior to be recorded within the general class of behaviors (e.g., ventral hold, adjusts feeding, eye contact, touch/pat); the fourth digit indicates 
whether the entry is the onset (1) of behaviors defined as continuous (e.g., smile), the termination of those behaviors $(0)$, or the simple occurrence of behaviors defined as discrete events (e.g., touch, pat) (2). Each behavior code number is defined in a coding manual by label and by careful and precise objective description of the behavior.

\section{INTERFACE, ERROR CHECKS, AND DATA REDUCTION}

Following an observation session, the Datamyte is connected to a Teletype computer terminal with a phone modum (either a standard model in the homebase laboratory or a portable model at the field site) for transmission of data to the central computer facilities. The Datamyte manufacturer provides easily attached cables for this purpose.

Using TAURUS, the timesharing system at the University of Texas, data from the Datamyte are sent over phone lines to the central computer site (dual Control Data 6600/6400 system), where they are stored on magnetic tape. As data are transmitted, they are echo printed on a local Teletype.

Datamyte sends data lines in the format:

five-digit line number (in increments of 10)
space
four-digit behavior code
comma
five-digit time (in hundredths of a second)

The program (BABES) ${ }^{2}$ developed for our research program initially checks the input for obvious errors: (1) line number missing, (2) line number out of sequence, (3) line number too large, (4) behavior code missing or zero, (5) behavior code not four digits long, and/or (6) time out of sequence.

After the observation record for a 10-min session has been read, the program sorts the data by behavior code, and then, within each behavior code block, sorts the data by the time of entry. Further error checking takes place as follows: (1) Check that all codes end in 0,1 , or $2 ;(2)$ check that all behavior codes ending in 1 (start of a behavior) have a corresponding code ending in 0 (end of a behavior); (3) if there are any start codes without a stop, check that the code terminates after the 10 -min observation limit. If so, terminate the code at the 10-min mark; if not, note as an error. A complete summary of all errors is printed, including location of and reason for the error. The corrections for errors are then edited into the behavior record using a Teletype or a CRT and an editing program provided by the computer center. When no errors remain in the record, a complete frequency count of all codes is prepared, printed, and stored. In addition, for all codes indicating the start of a continuous variable, a cumulative duration for the code is derived using the start codes, the termination codes, and the recorded times.
Thus, a permanent file is created for each parentinfant pair which includes the corrected (edited) observation record, the total frequency of each parent and infant behavior variable, and the total duration of continuous behavior variables. The final stage of data reduction involves the calculation of conditional and baseline probability indices of the contingencies between parent and infant behaviors.

\section{DATA ANALYSIS: CONDITIONAL AND BASELINE PROBABILITIES}

The conditional and baseline probability program $(C B P)^{3}$ is designed to calculate a set of conditional probabilities and baseline probabilities (i.e., expected value) for each behavioral variable specified by the user. For the conditional probabilities, two behavioral events are specified: (1) the conditional event (e.g., a potentially eliciting or inhibiting behavior by one member of the dyad such as an infant distress signal) and (2) the criterion behavior (e.g., a potential parental response such as soothing the infant). In addition, a conditional time interval (i) is specified to establish a time-lag limit (e.g., 3, 6, 9, etc. sec) for the occurrence of the possible criterion events that follow the conditional event. One may choose: (1) an infant behavior, say, $A$, to be the conditional event; (2) any parent behavior, say, B, to be the criterion event; (3) a conditional time interval of $9 \sec (i=9)$. For each dyad the CBP program calculates the conditional probability of parent behavior $B$ given the occurrence of infant behavior $A$, that is, the probability of the parent behavior (B) occurring within $9 \mathrm{sec}$ following the occurrence of the infant behavior (A). The onset of an interval is established by the occurrence of the infant behavior (A) and the termination of the interval is at the end of $i=9 \mathrm{sec}$. The formula is:

$$
P_{i}(B \mid A)=\frac{P_{i}(A \rightarrow B)}{P_{i}(A)}
$$

where $\mathbf{P}_{\mathbf{i}}(\mathbf{B} \mid \mathbf{A})$ is read: "The conditional probability of the occurrence of event $B$ within a time interval of length $i$ following the occurrence of event $A$." The value of $P_{\mathbf{i}}(\mathbf{B} \mid \mathbf{A})$ is calculated as:

$$
P_{i}(B \mid A)=\frac{f_{i}(A \rightarrow B)}{f_{i}(A)}
$$

where $f_{i}(A \rightarrow B)$ is the frequency of intervals whose onset is determined by the occurrence of $A$ in which $B$ followed $A$ within $i$ sec. The term $f_{i}(A)$ is the total frequency of $i$-sec intervals defined by the occurrence of $A$ (i.e., the total frequency of A). Note that the i-sec intervals may overlap. These conditional probabilities provide an estimate of the temporal contingency between the criterion behaviors and the conditional events. However, the conditional probability estimates are meaningful only in relation to the expected value or 
baseline probabilities for the criterion events. Thus, the baseline probabilities are also calculated. The formula for the baseline probability of $B$ is:

$$
P(B)=\frac{f_{i}(B)}{N_{f_{i}}}
$$

where $f_{i}(B)$ is the frequency of $i-\sec$ intervals across the entire behavior record for a given dyad in which $B$ occurred and $\mathbf{N}_{f_{i}}$ is the total number of $i$-sec intervals in the behavior record. For baseline probability calculations, the entire behavior record is segmented into sequential $\mathbf{i}$-sec intervals; thus, $\mathbf{N}_{\mathbf{f}_{\mathbf{i}}}=$ (total time) $/ \mathbf{i}$.

Input for $\mathrm{CBP}$ is the time-line data record from permanent files as they were collected with the Datamyte and edited. The user specifies the conditional behavior (A) that defines the onset of the time intervals, and the user specifies the length of the time intervals. The CBP program then forms the time intervals from the Datamyte input record. Each defined interval begins with the conditional behavior and includes all other behaviors which follow the conditional behavior within the specified time period (i.e., i). Subsequent to the formation of the time intervals, the interval frequencies [e.g., $\left.f_{i}(A \rightarrow B)\right]$ are counted and the conditional probabilities calculated for all possible criterion behaviors.

The CBP program then forms the time intervals in the behavior record necessary for the calculation of the baseline probabilities for all of the criterion behaviors. The intervals are formed by segmenting the entire observational period into equal-length intervals (i), where the length of the intervals is the same as that used to determine the conditional probabilities. Interval frequencies [e.g., $f_{i}(B)$ ] are then counted and used to calculate the baseline probabilities. The output from CBP consists of: (1) the number of intervals in which $B$ followed $A$ in the intervals defined by the occurrence of $A$, (2) the total number of intervals defined by the occurrence of $A,(3)$ the conditional probability of $B$ given that A occurred, (4) the number of intervals in which B occurred, out of the total number of possible intervals, (5) the total number of possible intervals, and (6) the baseline probability that $\mathrm{B}$ will occur in any given interval. The output is stored on permanent files.

The CBP program, written in FORTRAN IV, offers several options. The user can specify any number of conditional events, any number of criterion behaviors, and any time interval length that is a multiple of .6 of a sec.

\section{SAMPLE CONDITIONAL PROBABILITY DATA}

For purposes of this paper, preliminary analyses were conducted on a single parent behavior variable to demonstrate the use of the data from the observation and coding procedures described above. The sample consists of the first 43 mothers and infants observed to date in the hospital setting. The behavior chosen for analyses was the mother's adjusting of the position in which she was holding the infant during feeding and play activities. Comparisons among the three ethnic groups of the frequency of adjusting holding patterns revealed no substantial differences between Black, Anglo, and Mexican-American mothers. However, a different pattern of results was obtained when this same behavior was analyzed as a criterion response to the conditional infant behavior labeled "negative vocalization" (fret, fuss). This approach involves the analysis of conditional probability estimates of the contingency with which mothers respond to infant fussing by adjusting the position in which they hold the infant. The conditional probability of mothers adjusting a hold, given that the infant has fussed (i.e., within 9 sec following fussing), and the baseline probability of this maternal behavior are the data utilized in this analysis of maternal responsiveness.

The conditional and baseline probabilities calculated by CBP were analyzed by ANOVA as a repeated measures factor, along with the classification variables of ethnicity, sex of infant, and context (feeding vs. playing). The analysis yielded a significant conditional vs. baseline probability main effect, showing that the temporal contingency between infant fussing and the mother's "adjust hold" responses were significant. A significant ethnicity of mother by conditional vs. baseline probability interaction revealed that the contingency of responding to negative vocalizations was not the same for all ethnic groups. Black mothers were more likely to adjust the way they held their infants after a negative vocalization than were either Mexican-American or Anglo mothers.

While the frequency analysis indicates that there are no differences among ethnic groups in the total number of times mothers adjust the way they hold their babies, the conditional probability analysis suggests that, after an infant distress signal has occurred, Black mothers are more apt to adjust their hold than are other mothers. This type of information warrants consideration of the Datamyte (and other similar time-based recording devices) as a valuable and essential tool for researchers interested in addressing similar issues.

\section{REFERENCE}

Torgerson, L. Datamyte 900. Behavior Research Methods \& Instrumentation, 1977, 9, 405-406.

\section{NOTES}

1. Earlier versions of this coding system were developed in association with Ross D. Parke and Candice W. Collmer at Fels Research Institute, Yellow Springs, Ohio.

2. Andrew Bell, Department of Psychology, University of Texas at Austin, wrote the BABES program.

3. Co-author Leitner wrote the CBP program.

\footnotetext{
(Received for publication July 5, 1977;
} revision accepted August 25, 1977.) 